\title{
Edema facial como manifestación inicial atípica de arteritis de células gigantes: a propósito de un caso y revisión de la literatura
}

\author{
Facial edema as an atypical initial manifestation of giant cell arteritis: a case report \\ and review of the literature
}

Karime Sabat ${ }^{1}$, Cecilia González ${ }^{1}$, Maria Jesús Garchitorena ${ }^{1}$, Homero Gac ${ }^{2}$, María Jesús Silva ${ }^{2}$

\begin{abstract}
Resumen
Introducción: la arteritis de células gigantes es la vasculitis de vaso grande más frecuente y se ve predominantemente en adultos mayores de 50 años. El diagnóstico es en base a la clínica que se compone de cefalea, polimialgia reumática, sensibilidad en relación a la arteria temporal, compromiso del estado general, entre otras cosas, más laboratorio que se evidencia reactantes de fase aguda elevados y anemia y se confirma con biopsia de arteria temporal. Métodos: reporte de un caso de una paciente con debut con cefalea y aumento de volumen facial, lo que conllevó un diagnóstico erróneo de celulitis facial. Debido a esta presentación atípica, se retrasó el diagnóstico de arteritis de la temporal y tratamiento oportuno. Resultados: la arteritis de células gigantes es una patología que posee un gran rango de presentaciones atípicas, lo que ocurre en hasta un $38 \%$ de los pacientes que poseen la enfermedad, manifestaciones que incluyen neuralgia del trigémino, infartos linguales, aneurismas aórticos, edema facial, entre otros. Conclusión: es muy relevante conocer las presentaciones atípicas de esta patología que son muy frecuentes de encontrar en los pacientes y conocerlas nos permite aumentar nuestra sospecha clínica permitiendo un diagnóstico y tratamiento oportuno, evitando consecuencias irreversibles por el retraso diagnóstico.
\end{abstract}

Palabras clave: arteritis de la temporal; arteritis de células gigantes; edema facial; manifestaciones atípicas; vasculitis; vasculitis atípicas; reumatología en geriatria.

\begin{abstract}
Introduction: giant cell arteritis is the most frequent large vessel vasculitis and is seen predominantly in adults over 50 years. The diagnosis is based on the clinic that is composed of headache, polymyalgia rheumatic, sensitivity near the temporal artery, compromise of the general condition, among other things, added to a laboratory that is evidenced like severe acute phase reactants and anemia and finally, is confirmed with temporal artery biopsy. Methods: a case report of a patient who debuted with headache and increased facial volume that led to a wrong diagnosis of facial cellulite. Because of this atypical presentation of the disease, the diagnostic took more time than usual and delayed the accurate diagnosis and timely treatment; this could have caused irreversible consequences. Results: giant cell arteritis has a wide range of atypical presentations; this may occur even up to $38 \%$ of patients that have this disease; manifestations include: trigeminal neuralgia, lingual infarct, aortic aneurysm, facial edema, and other symptoms. Conclusion: it is important to study the atypical presentations of this pathology because they are usually founded in patients. If we are informed about the atypical presentations, we can increase our clinical suspicion, and that allows us to get the right diagnosis and opportune treatment, avoiding irreversible consequences because of a late diagnosis.
\end{abstract}

Keywords: temporal arteritis; giant cell arteritis; facial edema; atypical manifestations; vasculitis; atypical vasculitis; rheumatology in geriatrics.

Fecha de envío: 5 de mayo de 2020 - Fecha de aceptación: 16 de junio de 2020

(1) Facultad de Medicina, Pontificia Universidad Católica de Chile

(2) Departamento de Geriatría, Pontificia Universidad Católica de Chile

Autor de correspondencia: mjgarchitorena@uc.cl 


\section{Introducción}

La arteritis de células gigantes (ACG), o la anteriormente denominada arteritis de la temporal, es la vasculitis de vaso grande más frecuente y suele verse en adultos mayores de 50 años, con predilección por el sexo femenino, además de ser infrecuente en raza negra. La ACG afecta predominantemente vasos grandes con preferencia por la aorta y sus ramas tanto extracraneales como intracraneales, siendo el primer grupo lo más clásico, típicamente la afección de las ramas de la carótida, en especial la arteria temporal (Longo et al., 2018). A la histología el hallazgo clásico es una panarteritis con infiltrados inflamatorios de células mononucleares dentro de la pared del vaso, con frecuente formación de células gigantes, habiendo proliferación de la íntima y fragmentación de la lámina elástica interna de las arterias (Winckler \& True, 2018).

Esta inflamación arterial crónica resulta en daño vascular como estenosis, oclusión e incluso aneurismas, lo que se manifiesta en los órganos involucrados como isquemia de los vasos afectados. Esto provoca junto con la inflamación, las diferentes manifestaciones típicas de la enfermedad como cefalea, polimialgia reumática asociada en un $40-50 \%$, sensibilidad en relación a la arteria temporal y su palpación, claudicación mandibular, sensibilidad del cuero cabelludo, síntomas oculares como repentina pérdida de visión transitoria o permanente, diplopia, entre otros (Vodopivec \& Rizze, 2018) y síntomas consuntivos como compromiso del estado general, fatiga, anorexia, baja de peso, sudoración y artralgias. Dentro de los exámenes de laboratorio, lo más frecuente es que únicamente tengan alteración de parámetros inflamatorios (VHS y PCR) y anemia (Longo et al., 2018).

El diagnóstico de la ACG es en base a la clínica, exámenes de laboratorio que demuestran una elevación en los reactantes de fase aguda y confirmación a través de la biopsia de arteria temporal que demuestra una vasculitis con infiltrado inflamatorio mononuclear (Nesher, 2014). Pese a esto, existe un 15\% de los pacientes que presentan biopsia negativa y en aquellos el diagnóstico puede facilitarse repitiendo la biopsia de forma bilateral o con imágenes como Ecografía Doppler que demuestra un halo hipoecogénico periluminal que representa el edema de la pared y es altamente sensible y específico de ACG, sin embargo, requiere de un operador entrenado. Actualmente, se está incluyendo en el diagnóstico por imágenes la Resonancia Magnética con contraste de alta resolución de las arterias temporales que plantea ser altamente sensible en visualizar la inflamación de la pared arterial, la Angiografía por tomografía de la aorta y sus vasos cuando el cuadro compromete grandes vasos (Nesher, 2014.), PET con 18-FDG que ha demostrado compromiso de grandes vasos como aorta y subclavia en un $50-80 \%$ de estos pacientes (Blockmans, 2012) y finalmente, la tomografía computada (TC) que permite ver la extensión de la enfermedad (Blockmans, 2012).

Sin embargo, a pesar de las múltiples herramientas disponibles para apoyar el diagnóstico, este en muchas ocasiones se hace difícil debido al variado espectro de manifestaciones que puede tener la ACG, llevando a procesos diagnósticos tórpidos con consecuente retraso en el tratamiento como sucedió en el caso que se presentará a continuación.

\section{Caso clínico}

Paciente de 72 años, previamente sana, autovalente, no usuaria de medicamentos diarios, sólo eszopiclona 1/3 de comprimido en caso de necesidad. En sus antecedentes quirúrgicos destacan 2 resecciones de carcinoma basocelular, no ha tenido hospitalizaciones recientes, no consume drogas y no tiene alergias.

Consulta al SU por cuadro de dos semanas de evolución de cefalea, asociada a aumento de volumen facial, dolor cervical y rigidez de cuello leve. En ese contexto se diagnostica una celulitis facial, con parámetros inflamatorios elevados, para lo cual se decide manejo con antibiótico endovenoso y oral, presentando inicialmente buena respuesta clínica, sin embargo, evoluciona con aumento de parámetros inflamatorios durante terapia con antibiótico oral.

Posteriormente se pesquisó dolor en molar y se realizó extracción dental por sospecha de infección. Por persistencia del cuadro, se realiza TAC de cavidades paranasales que demuestra inflamación en tejido blando maxilar superior izquierdo y RNM macizo facial con hallazgos compatible con perineuritis óptica bilateral (Figura 1) y cambios postquirúrgicos de los senos maxilares. Se decide traslado a centro de mayor complejidad para continuar estudio y manejo por sospecha de Arteritis de células gigantes.

Ingresa en buenas condiciones generales, al interrogatorio dirigido sin historia de baja de peso, fiebre, dolor o claudicación mandibular ni cintura escapular, sin cefaleas previa. Dado sospecha de ACG, se decide inicio de terapia con prednisona $60 \mathrm{mg} /$ día. Se solicita estudio con ANA (-) anti DNA (-) ANCA (-) perfil ENA (-) RPR NR, Toxoplasma (-) y se realiza biopsia de arteria temporal derecha que impresiona con inflamación crónica importante.

Diagnóstico anatomopatológico de la biopsia: presencia de moderado infiltrado inflamatorio linfoplasmocitario, localizado predominantemente en la capa media, con numerosas células gigantes multinucleadas. Sin focos supurados ni necrosis de caseificación. Hallazgos morfológicos compatibles con arteritis de células gigantes. 
Evaluada por reumatología, con cuadro compatible con ACG por lo que se indica mantener terapia corticoidal hasta control al alta.

Paralelamente es evaluada por oftalmología, con agudeza visual sin mayor compromiso (no característico de ACG), por lo que se indica completar estudio con nueva resonancia, se realiza RNM de órbita que muestra perineuritis óptica bilateral (Figura 2). Por sospecha de que esto estuviera en contexto infiltrativo y no por el cuadro de ACG, se realizó estudio de LCR para descartar linfoma, lo cual arrojó un citoquímico normal e lgG en LCR en rango normal. Esto hace menos probable una enfermedad linfoproliferativa u otra afección inmunológica del sistema inmunológico. Se continúa terapia antibiótica con ceftriaxona por periodontitis con buena respuesta, completa 5 días. Paciente evoluciona favorablemente, sin cefalea, en buenas condiciones generales con disminución de parámetros inflamatorios, por lo que se decide alta con control ambulatorio. En control ambulatorio a los tres meses post alta, la paciente declara no presentar nuevas crisis de cefalea o edema facial, ni otros síntomas que sugieran recidiva, con mejoría del estado general, por lo que se mantiene terapia corticoesteroidal oral.

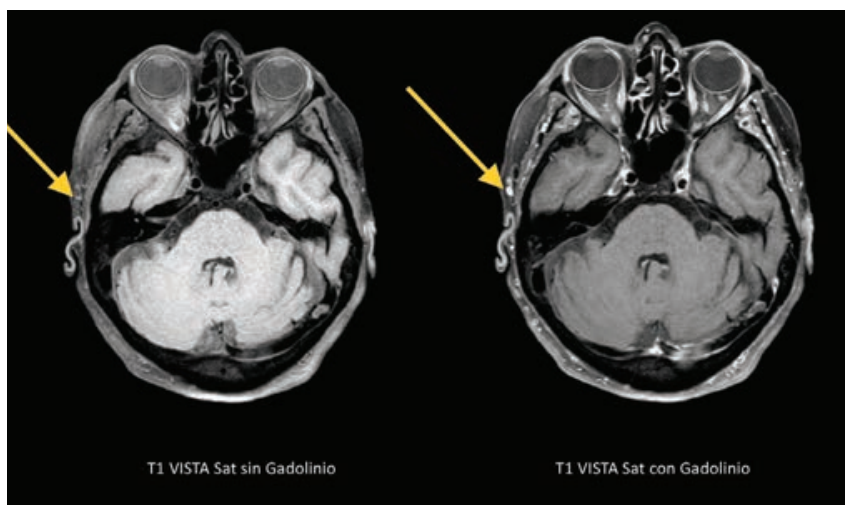

Figura 1: RNM de macizo facial. Engrosamiento parietal de trayecto de arteria temporal superficial derecha

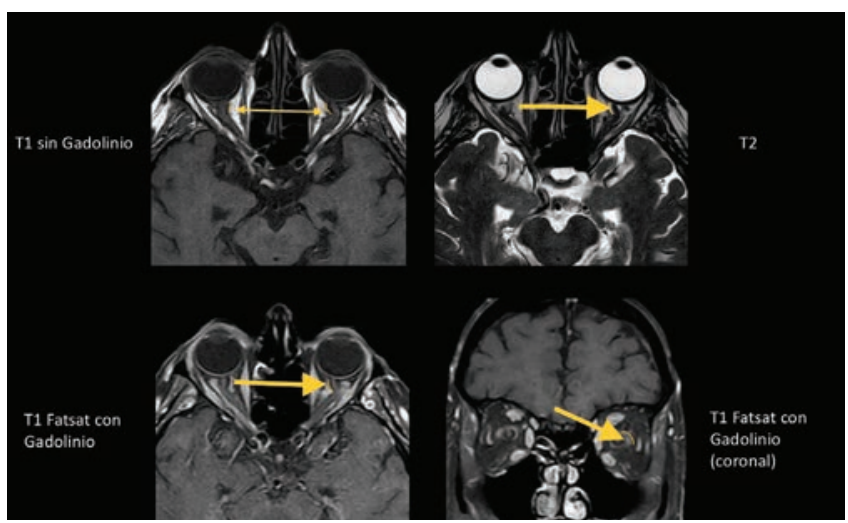

Figura 2: RNM de óptica bilateral. Engrosamiento de la vaina perineural óptica bilateral, excéntrica

* Paciente otorga consentimiento informado de manera voluntaria para publicación de caso clínico.

\section{Discusión}

Frente a un caso de arteritis de células gigantes, en la literatura están descritos múltiples signos y síntomas que apoyan al diagnóstico de ésta por estar frecuentemente presentes en el cuadro clínico. Dentro de los síntomas más clásicos destacan: cefalea 70-80\%, sensibilidad en el cuero cabelludo $20-40 \%$, sensibilidad o aumento de volumen en relación a la arteria temporal 30-60\%, claudicación mandibular en un 30-40\%, síntomas oculares en 15-45\% (pérdida de visión transitoria o permanente y diplopia), fiebre, compromiso del estado general y baja de peso $30-60 \%$, y polimialgia reumática en $20-60 \%$ (Carro A., 2013). Todos estos síntomas junto con alteraciones de laboratorio como elevación de PCR, VHS y presencia de anemia, ayudan al diagnóstico.

Pese a esto, el diagnóstico de ACG no siempre se da de forma fácil ya que un porcentaje significativo de los pacientes no presentan el cuadro clínico clásico explicado previamente si no que manifestaciones atípicas de la enfermedad que se describen en un 5-38\% de los casos (Rahman \& Rahman, 2005). Dentro de estas, las más frecuentemente descritas son: accidentes cerebrovasculares, accidentes isquémicos transitorios, mono o polineuropatía periférica en brazos y piernas, síntomas vestibulococleares como pérdida de audición, tinnitus, vértigo, infartos de lengua o cuero cabelludo, síndrome de arco aórtico, insuficiencia aórtica, aneurisma aórtico, síntomas respiratorios como tos, dolor y edema facial, neuralgia del trigémino, odinofagia y disfonía (Carro, 2013; Imran et al., 2015).

En éste caso clínico en particular, es interesante analizar que la paciente presentaba un cuadro poco característico de ACG que conllevo a un retraso en su diagnóstico y tratamiento ya que del cuadro típico únicamente presentaba cefalea, dolor cervical y aumento de parámetros inflamatorios, sin embargo, las manifestaciones atípicas que presentaba como aumento de volumen facial, que ha sido descrito en la literatura como una forma de presentación inusual, sin embargo, en una serie prospectiva de 345 pacientes con esta patología se evidenció en una frecuencia de $12 \%$ de ellos (Letellier et al., 2004; Liozon et al., 2006) y ha sido evidenciado en múltiples casos clínicos (Anthony et al., 2008) y dolor en pieza dental, que pudiese estar en contexto de una neuralgia del trigémino o claudicación mandibular no característica. Esto conllevó un amplio diagnóstico diferencial incluyendo celulitis y periodontitis que provocó una terapia antibiótica y extracción de pieza dental que podría haberse evitado.

Lo valioso de este caso, es que nos otorga una perspectiva más amplia del cuadro de ACG ya que este no siempre se presenta de forma clásica, pues en la práctica clínica lo que se puede 
evidenciar es que presenta un amplio espectro de manifestaciones. Es por esto, que requiere un alto nivel de sospecha ya que la ACG es una emergencia médica puesto que los pacientes necesitan un tratamiento oportuno basado en glucocorticoides orales de $40 \mathrm{a}$ $60 \mathrm{mg} / \mathrm{día}$, dosis altas de glucocorticoides endovenosos en caso de riesgo inminente de ceguera como cuando se presentan con amaurosis fugax tratado con Metilprednisolona de $500 \mathrm{mg}$ a 1 gramo endovenoso por tres días seguido de terapia oral con prednisona de 1 mg/kg/día (Salvarani et al., 2008; Bienvenu et al.,2016) y, en algunos casos, uso crónico de aspirina en dosis bajas ( $\leq 100 \mathrm{mg} /$ día), que si bien es controvertido, la evidencia nos permite concluir que debe ser prescrito considerando los riesgos y beneficios de cada paciente $y$, especialmente siguiendo las recomendaciones corrientes de prevención de complicaciones ateroscleróticas (Bienvenu et al.,2016). En caso de no brindar tratamiento esta patología trae por consecuencia complicaciones irreversibles producto de la inflamación de los vasos como la ceguera permanente, accidente cerebrovascular y aneurismas de la aorta torácica y abdominal.

Finalmente, este caso presenta una peculiaridad ya que en la resonancia magnética de órbita se evidenció una perineuritis óptica bilateral, hallazgo no reportado hasta el momento en la literatura como una alteración oftalmológica que pueda tener correlación con ACG. Lo cual es especialmente relevante, ya que la complicación oftalmológica más común en la ACG corresponde a la neuritis óptica isquémica anterior (NOIA), que por su presentación se configura como una emergencia médica. Sin embargo, se destaca que esta última presenta una clínica bastante florida con pérdida de visión asociada a evidencias de hemorragias papilares al fondo de ojo, además de manchas algodonosas y palidez del disco óptico, que puede conllevar a secuelas permanentes (Liu et al., 2012). En este contexto queremos destacar el hallazgo en la RNM de la paciente, ya que estimamos podría corresponder a una alteración de perfil asintomático relacionada con la NOIA.

\section{Conclusión}

La ACG es una patología donde los pacientes no siempre presentarán los clásicos síntomas descritos como prevalentes y pueden debutar con un cuadro incluso oligosintomático. Por otra parte, no es infrecuente que las manifestaciones del cuadro sean atípicas, por lo que debemos conocerlas para poder sospechar una ACG.

Los exámenes de laboratorio e imágenes pueden apoyar nuestra sospecha diagnóstica frente a cuadros menos clásicos de la patología.

La ACG es un cuadro de gravedad que puede comprometer y secuelar en forma importante a los pacientes, por lo que frente a su sospecha se debe iniciar tratamiento en forma inmediata.

\section{Conflictos declarados por los autores}

Se declara no tener conflictos de interés por parte de los autores en el desarrollo de este caso clínico.

\section{Referencias}

Anthony J, Dennis J, Janusz J, Suzanne HDO \&John R. (2008). Allergy Asthma Proceedings 29,538-550.

Bienvenu B, Ly KH, Lambert M, Agard C, André M, Benhamou Y, Bonnotte B, de Boysson H, Espitia O, Fau G, Fauchais AL, Galateau-Sallé F, Haroche J, Héron E, Lapébie FX, Liozon E, Luong Nguyen LB, Magnant J, Manrique A, Matt M, de Menthon M, Mouthon L, Puéchal X, Pugnet G, Quemeneur T, Régent A, Saadoun D, Samson M, Sène D, Smets P, Yelnik C, Sailler L, Mahr A et al.,. (2016). Management of giant cell arteritis: Recommendations of the French Study Group for Large Vessel Vasculitis (GEFA). La Revue de Médicine Interne 37, 154-65.

Blockmans D. (2012) Diagnosis and extension of giant cell arteritis. Contribution of imaging techniques. La Presse Médicale 41, 948-954.

Carro A, Lozano A \& Castellano J.(2013). Enfermedades reumáticas: actualización SVR. 10th ed. España.

Imran T \& Helfgott S .(2015). Respiratory and otolaryngologic manifestations of giant cell arteritis. Clinical and Experimental Rheumatology online 33.

Letellier P, Andrés E \& Dassonville L. (2004). Edème de la face et maladie de Horton : étude monocentrique prospective portant sur 345 patients. La Revue de Médicine Interne.

Liu GT, Volpe NJ, Galetta SL \& WB Saunders. (2012). Visual loss: optic neuropathies. In Neuro-Ophthalmology:Diagnosis and Management, pp 101-196. Elsevier

Liozon E, Ouattara B, Portal MF, Soria P, Loustaud-Ratti V \& Vidal E. (2006). Head-and-neck swelling: an under-recognized feature of giant cell arteritis. A report of 37 patients. 24, S20-5.

Longo D, Fauci A, Jameson L \& Loscalzo J (2018). Harrison's Principles of Internal Medicine. 20th ed. United States of America.

Nesher G. (2014) The diagnosis and classification of giant cell arteritis. Journal of Autoinmunity 48, 73-75.

Rahman W \& Rahman F. (2005). Giant Cell (Temporal) Arteritis: An Overview and Update. Survey of Ophthalmology: 50, 415-428. 
Sabat et al.

Salvarani C, Cantini F \& Hunder GG. (2008). Polymyalgia rheumatica and giant-cell arteritis. Lancet: 372, 234-45.

Vodopivec I \& Rizzo J. (2018). Ophthalmic manifestations of giant cell arteritis. Rheumatology: 57, ii63-ii72.
Winkler A \& True D. (2018). Giant Cell Arteritis Review. Missouri medicine: 115, 468-470. 